\title{
ARTICLE
}

Molecular Diagnostics

\section{L1CAM further stratifies endometrial carcinoma patients with no specific molecular risk profile}

Felix KF Kommoss ${ }^{1}$, Anthony N. Karnezis ${ }^{2}$, Friedrich Kommoss ${ }^{3}$, Aline Talhouk ${ }^{2}$, Florin-Andrei Taran ${ }^{4}$, Annette Staebler ${ }^{5}$, C. Blake Gilks ${ }^{6}$, David G. Huntsman ${ }^{2}$, Bernhard Krämer ${ }^{4}$, Sara Y. Brucker ${ }^{4}$, Jessica N. McAlpine ${ }^{7}$ and Stefan Kommoss ${ }^{4}$

BACKGROUND: The newly developed Proactive Molecular Risk Classifier for Endometrial Cancer (ProMisE) has consistently been shown to be prognostically significant in endometrial carcinomas (EC). Recently, we and others have demonstrated L1 cell-adhesion molecule (L1CAM) to be a significant indicator of high-risk disease in EC. In the current study, it was our aim to determine the prognostic significance of aberrant L1CAM expression in ProMisE subgroups in a large, single centre, population-based EC cohort. METHODS: ProMisE (POLE; MMR-D; p53 wt/NSMP; p53 abn) classification results from a cohort of 452 EC were available for analysis. L1CAM expression was studied by immunohistochemistry on whole slides. Correlations between clinicopathological data and survival were calculated.

RESULTS: Expression of L1CAM was most frequent in p53 abnormal tumours (80\%). L1CAM status was predictive of worse outcome among tumours with no specific molecular profile (p53 wt/NSMP) $(p<0.0001)$. Among p53 wt/NSMP EC, L1CAM remained a significant prognosticator for disease-specific survival after multivariate analysis $(p=0.035)$.

CONCLUSION: L1CAM status was able to significantly stratify risk among tumours of the large p53 wt/NSMP ProMisE subgroup of EC. Furthermore, our study confirms a highly significant correlation between mutation-type p53 immunostaining and abnormal L1CAM expression in EC.

British Journal of Cancer (2018) 119:480-486; https://doi.org/10.1038/s41416-018-0187-6

\section{BACKGROUND}

Endometrial cancer (EC) is the most common gynecological malignancy in women in the western world. ${ }^{1} \mathrm{EC}$ patient treatment is currently based on risk classification, for which clinicopathological criteria, such as histological type, FIGO grade and stage are key parameters. $^{2-5}$ However, several studies have shown the evaluation of pathological features such as grade and histotype to be of only limited reproducibility, particularly in high-grade tumours. $^{6-8}$ Over the past years, molecular studies have looked into identifying more reproducible prognosticators in EC with the aim of creating a better risk classification system.

An important step has been the genomic characterisation of EC by The Cancer Genome Atlas (TCGA) group and others, defining four prognostically distinct molecular subgroups. ${ }^{9,10}$ In order to simplify the methodologies and to lower cost involved in identifying these molecular subgroups, research teams have recently described surrogate markers that can be studied more easily. ${ }^{11-15}$ Talhouk et al. have developed the Proactive Molecular Risk Classifier for Endometrial Cancer (ProMisE), demonstrating an easy pragmatic classification system for ECs. ${ }^{11,12,15}$ The four prognostic ProMisE subgroups are as follows: DNA Polymerase epsilon exonuclease domain mutation (POLE), mismatch repair deficiency (MMR-D), p53 wild-type (p53 wt) and p53 abnormal (p53 abn). ${ }^{11}$ Tumours within the p53 wt group, which do not harbour mutations of neither POLE, mismatch repair genes nor abnormal p53, are also referred to as tumours with "No Specific Molecular Profile, NSMP". ${ }^{16}$

The L1 neuronal cell-adhesion molecule (L1CAM/CD171) has recently gained attention as a specific prognosticator and potential therapeutic target in EC and other tumours. ${ }^{17}$ Multiple studies have shown the prognostic significance of L1CAM immunohistochemistry (IHC) in large cohorts of EC. ${ }^{18-23}$ The upregulation of L1CAM was found to be a major driver for tumour cell motility and to be closely associated with the process of epithelial to mesenchymal transition (EMT). ${ }^{24-28}$ Tumours showing histological evidence of EMT are frequently biologically aggressive neoplasms and tend to present at an advanced tumour stage. The downregulation of E-Cadherin and hormonal receptors, and the upregulation of L1CAM have been described as main features of EMT in EC. ${ }^{29,30}$ Furthermore, multiple studies depict a connection between L1CAM expression and type II EC histology (serous/clearcell histotype), known to display high rates of abnormal p53 status. ${ }^{18,19,31}$ So far, specific regulations of L1CAM expression on a transcriptional level have remained enigmatic. Recent reports

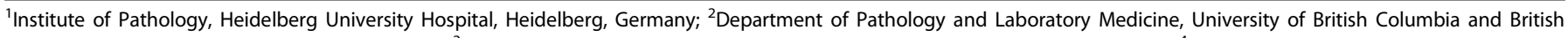

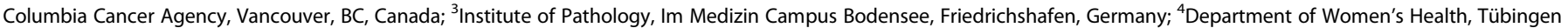

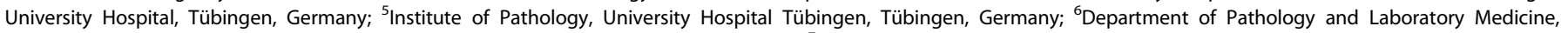

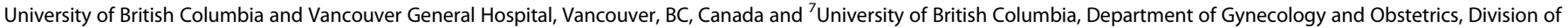
Gynecologic Oncology, Vancouver, BC, Canada

Correspondence: Stefan Kommoss (stefan.kommoss@med.uni-tuebingen.de)

Received: 9 April 2018 Revised: 18 June 2018 Accepted: 25 June 2018

Published online: 27 July 2018 
have suggested a strong association between L1CAM expression and mutant-type p53 immunoreactivity; however, there is also evidence suggesting a p53-independent mechanism of L1CAM expression in multiple molecular subgroups. ${ }^{31,32}$

Recently, Karnezis et al. correlated L1CAM and other immunohistochemical markers with ProMisE subgroups in a population-based cohort of EC and found high L1CAM expression to be highly associated with tumours showing mutationtype p53 expression and to identify poor prognosis tumours within MMR-D and p53 wt molecular subgroups. ${ }^{31}$ Stelloo et al. found similar results in a large, non-population-based cohort of EC patients (PORTEC). ${ }^{14}$

The aim of our study was to investigate the correlation between ProMisE subgroups and pathological L1CAM expression in a large, single centre, population-based cohort. Specifically, we wanted to study the potential of L1CAM to improve molecular EC classification, especially within the intermediate-risk molecular subgroups (MMR-D and p53wt/NSMP). We discuss possible mechanisms by which L1CAM expression may lead to aggressive clinical behaviour in EC.

\section{METHODS}

Study cohort

Patients treated for primary EC at the University Hospital Tübingen between 2003 and 2013 were identified and clinical data, tissue samples and specialised gynecopathological review data were collected. Risk classification was performed applying current ESMO 2016 criteria. $^{2}$ Patients with synchronous or metachronous second malignancies were excluded. Follow-up data was obtained from the Tübingen University Hospital Clinical Cancer Registry and subsequently updated, allowing for evaluation of overall survival (OS) and disease-specific survival (DSS). The Tübingen University Independent Ethics Committee issued study approval.

\section{Molecular classification}

The ProMisE classification system applied in this study has previously been described in detail. ${ }^{15}$ Briefly, tumours were evaluated for 4 prognostic subgroups: Polymerase epsilon exonuclease domain mutations (POLE) detected through sequencing (exon 9-14), mismatch repair deficiency (MMR-D) evaluated using IHC for the absence of MSH6 or PMS2, and TP53 mutation evaluated using IHC for p53, revealing p53 wild-type (p53 wt/ NSMP) and p53 abnormal (p53 abn) subgroups.

Immunohistochemistry

L1CAM IHC (Clone 14.10, Covance; 1:50 dilution) was performed according to established protocols. ${ }^{19}$ L1CAM expression was scored according to percentage of positivity in tumour cells (score $0=0 \%$, score $1=1-10 \%$, score $2=>10-50 \%$ and score $3=>50 \%$; Supplementary Figure 1) and tumours were determined L1CAM positive, if $\geq 10 \%$ (score 2 and 3 ) of epithelial tumour cells showed membranous L1CAM staining. The threshold for L1CAM positivity ( $\geq 10 \%$ of tumour cells) was previously established by Zeimet et al., based on the cutoff that best correlated with prognosis. ${ }^{19}$ Evaluation of L1CAM IHC results was performed independently by two of the authors (FKFK, AS), who were blinded for outcome and ProMisE subgroups. In all cases with discrepant scoring results, a consensus was reached between both investigators.

Statistical analysis

Associations between L1CAM, ProMisE subgroups and other clinicopathological parameters were assessed using the fisher'sexact-test (FET). Survival analysis for OS and DSS were calculated using the Kaplan-Meier and cox-proportional hazard model. Pvalues (Likelihood-ratio test, LRT) and corresponding confidence intervals $(\mathrm{Cl})$ of $95 \%$ were recorded.

\section{RESULTS}

Study cohort and molecular classification

Four hundred fifty-two cases of primary EC were available for the current study. Patient follow-up data allowed for OS calculation in all 452 cases, and for DSS in 450 cases. Median follow-up time was 68 months (1-158 months). Study cohort characteristics, including ProMisE classification are given in Table 1 and are derived from previously published data. ${ }^{15} \mathrm{~A}$ small proportion of tumours (8 cases, 1.8\%) demonstrated more than one molecular feature (e.g. POLE and p53 abn, or POLE and MMR-D), and were classified as POLE or MMR-D, respectively, by strictly following the ProMisE decision tree, which dictates the order in which tumours are assigned to a specific molecular subgroup. ${ }^{15}$

L1CAM Immunohistochemistry

L1CAM IHC was performed in all 452 cases, of which results for ESMO non-high-risk tumours were previously reported. ${ }^{23}$ L1CAM was completely negative (score 0 ) in 320/452 (70.8\%) tumours, showed minimal staining (score 1, 1-10\% of tumour cells) in 35/ $452(7.7 \%)$, and positive staining ( $\geq 10 \%$ of tumour cells) in $97 / 452$ (21.5\%) tumours. Of positive tumours, 59/452 (13.1\%) showed moderate L1CAM staining (score 2, > 10-50\%), and 38/452 (8.4\%) showed strong L1CAM expression (score 3, > 50\%).

L1CAM and ProMisE molecular subgroups

Figure 1 illustrates L1CAM positivity rates within ProMisE subgroups. 7/42 (16.7\%) of tumours with POLE, 26/127 (20.5\%) of tumours with MMR-D, 19/228 (8.3\%) of tumours with p53 wt/ NSMP and $45 / 55$ (81.8\%) of tumours with p53 abn were L1CAM positive (Table 1; Fig. 1a, b). Inversely, ProMisE subgroups were distributed among L1CAM-positive tumours as follows: 7/97 (7.2\%) POLE, 26/97 (26.8\%) MMR-D, 19/97 (19.7\%) p53 wt/NSMP, and 45/ 97 (46.3\%) p53 abn (Fig. 1c). The differences in percentage of L1CAM positivity among ProMisE subgroups was statistically significant $(p<0.001)$.

Survival analysis of the whole cohort

Univariate survival analyses of L1CAM within each ProMisE subgroup (Table 2A) showed that L1CAM status had a statistically significant prognostic impact only among p53 wt/NSMP tumours. Here, L1CAM positivity was accompanied with a Hazard Ratio (HR) of $3.78(\mathrm{Cl}$ $1.69-7.61 ; p=0.002$ ) for OS and 7.82 (Cl 2.65-21.12; $p=0.0008)$ for DSS (Table 2A). Kaplan-Meier analyses showed significantly worse outcome for L1CAM positive, p53 wt/NSMP tumours with 5-year OS rates of $88.2 \%$ for L1CAM negative and $51.5 \%$ for L1CAM-positive tumours (Fig. 2c; $p<0.001$ ), and $95.5 \%$ for L1CAM negative and $65.5 \%$ for L1CAM-positive tumours for 5 -year DSS (Fig. 2d; $p<0.001$ ). For the remaining three subgroups, only a prognostic trend for L1CAM positivity and worse outcome was observed.

The p53 wt/NSMP subgroup was further stratified using L1CAM status in a univariate model (Table 3). Here the L1CAM-positive subgroup of p53 wt/NSMP tumours was at higher risk for fatal outcome, when compared to the p53 wt/NSMP, L1CAM negative subgroup with a HR of $6.94(\mathrm{Cl} 2.56-18.74 ; p<0.001)$ for DSS. In the same statistical model p53 abn tumours where also at higher risk for disease-specific death with a HR of 11.52 ( $\mathrm{Cl} 5.55-23.90 ; p<0.001)$.

In a multivariate analysis including ProMisE subgroups, age and L1CAM status (Table 2B), L1CAM status showed a trend toward prognostically significance for DSS; ProMisE subgroups were prognostically significant for OS and DSS. HR for L1CAM-positive tumours was $1.33(\mathrm{Cl} 0.77-2.22 ; p=0.3)$ for $\mathrm{OS}$ and $2.05(\mathrm{Cl}$ : $1.00-4.10 ; p=0.05)$ for DSS. There were relatively few L1CAMpositive tumours, which limited our power to study additional variables in the model using all four ProMisE subgroups.

p53 wt/NSMP subgroup analyses

Detailed statistical subgroup analyses were performed on the 228 p53 wt/NSMP tumours, which are summarised in Table 4 and 
Table 1. Clinicopathological data and L1CAM

\begin{tabular}{|c|c|c|c|c|}
\hline & Total & L1CAM negative & L1CAM positive & $P$ value \\
\hline \multicolumn{5}{|c|}{ Clinicopathological parameters } \\
\hline Mean $( \pm s d)$ & $60.2( \pm 11.5)$ & $63.8( \pm 11.6)$ & $69.3( \pm 10.1)$ & $<0.001$ \\
\hline Median & 65.3 & 64.1 & 69.8 & \\
\hline Median & 27.7 & 28 & 26.8 & \\
\hline Missing & 20 & 16 & 4 & \\
\hline \multicolumn{5}{|l|}{ ProMisE classification } \\
\hline POLE & $42(9.3 \%)$ & $35(9.8 \%)$ & $7(7.2 \%)$ & $<0.001$ \\
\hline MMR-D & $127(28.1 \%)$ & $101(28.5 \%)$ & $26(26.8 \%)$ & \\
\hline II-IV & $87(19.2 \%)$ & $52(14.7 \%)$ & $35(36.1 \%)$ & \\
\hline \multicolumn{5}{|l|}{ Tumour grade } \\
\hline Grade 1 & $282(62.4 \%)$ & $267(75.2 \%)$ & $15(15.5 \%)$ & $<0.001$ \\
\hline Grade 2 & $75(16.6 \%)$ & $58(16.3 \%)$ & 17 (17.5\%) & \\
\hline Grade 3 & $95(21 \%)$ & $30(8.5 \%)$ & $65(67 \%)$ & \\
\hline \multicolumn{5}{|l|}{ Histology } \\
\hline Endometrioid & $397(87.8 \%)$ & 349 (98.3\%) & $48(49.5 \%)$ & $<0.001$ \\
\hline Non-endometrioid & 55 (12.2\%) & $6(1.7 \%)$ & 49 (50.5\%) & \\
\hline \multicolumn{5}{|l|}{ LVSI } \\
\hline Negative & $388(85.8 \%)$ & $315(88.7 \%)$ & $73(75.3 \%)$ & 0.005 \\
\hline Intermediate & $64(14.1 \%)$ & $58(16.4 \%)$ & $6(6.2 \%)$ & \\
\hline High-intermediate & $27(6 \%)$ & $22(6.2 \%)$ & $5(5.2 \%)$ & \\
\hline High & $131(29 \%)$ & $62(17.4 \%)$ & 69 (71.1\%) & \\
\hline
\end{tabular}

Supplementary Table 1. Among p53 wt/NSMP tumours L1CAM positivity was significantly associated with high tumour grade (FIGO grade 3) and high FIGO stage (II-IV) (Supplementary Table 1). In a multivariate model only including factors available from preoperative biopsy or curettage samples (age, histotype, and FIGO grade), L1CAM status was a strong and independent prognosticator for DSS within the p53 wt/NSMP subgroup. HR for L1CAM positivity was $2.43(0.99-5.41 ; p=0.052)$ for OS and 3.80 (Cl: $1.10-12.16 ; p=0.035)$ for DSS (Table $4 \mathrm{~A}$ ). In a multivariate model including factors available from hysterectomy specimens [age, histotype, FIGO grade, FIGO stage, and lymphovascular space invasion (LVSI)], L1CAM status remained prognostic for DSS (HR of 4.03, $p=0.035$, Table 4B).

\section{DISCUSSION}

The recent introduction of clinically practical molecular classification systems of EC such as ProMisE is an important advancement in EC tumour classification by focusing on molecular parameters that have better reproducibility compared to the current WHO system, which is solely based on histomorphology. ${ }^{11,12,15}$ However, in order to guide surgery and/or adjuvant treatment there is still a need to further refine molecular risk stratification. This is most important for intermediate-risk groups of MMR-D and p53 wt/NSMP tumours, which constitute a large percentage of cases $(64-79 \%))^{11,12,15}$ Our study demonstrates that L1CAM expression correlates with high grade, high stage, and poor prognosis for OS and DSS within the p53 wt/NSMP subgroup of EC, which agrees with previous studies on similar cohorts. ${ }^{14,31}$

The fact that L1CAM expression in our study was prognostic in the intermediate-risk group of $\mathrm{p} 53 \mathrm{wt} / \mathrm{NSMP}$ tumours using both preoperative and postoperative variables indicates that the addition of LICAM IHC to the ProMisE (or similar) molecular classifier could add useful information for both prognosis and treatment (Table 4). The expression of L1CAM in p53 wt/NSMP endometrioid carcinoma on curettage specimens might indicate the need to perform more aggressive surgery due to the correlation of L1CAM with advanced stage, similar to p53 abn 
a

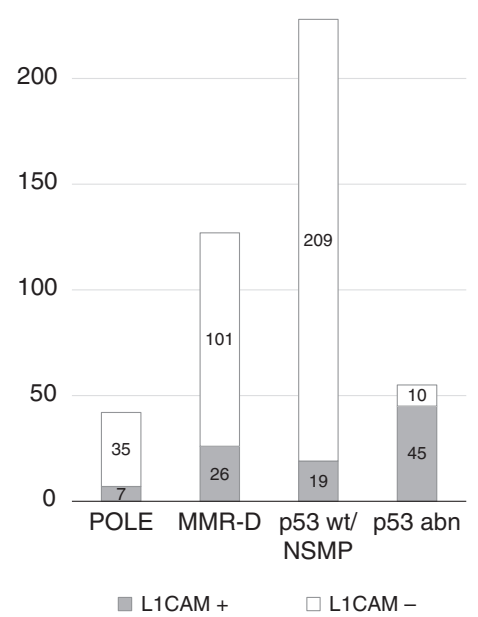

b

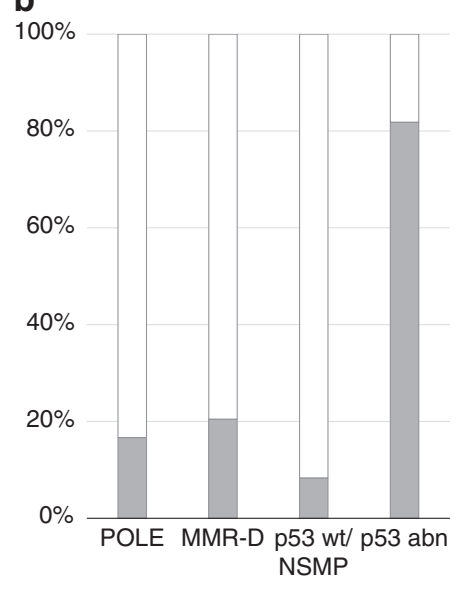

C

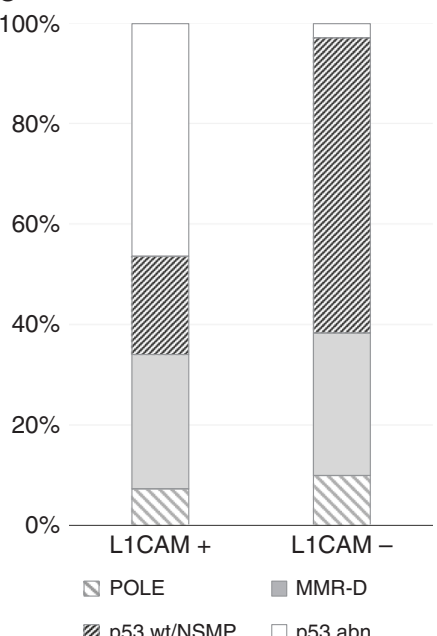

Fig. 1 L1CAM-positive cases among ProMisE subgroups: a Represented as absolute numbers b Represented as the percentage of each molecular subgroup. c Percentage of ProMisE subgroups among L1CAM positive and negative EC

\begin{tabular}{|c|c|c|c|c|c|c|}
\hline & & OS & & & DSS & \\
\hline \multicolumn{7}{|l|}{ A } \\
\hline \multicolumn{7}{|l|}{ POLE } \\
\hline \multicolumn{7}{|l|}{ P53 wt/NSMP } \\
\hline L1CAM negative (ref) & $41 / 228$ & - & & $16 / 227$ & - & \\
\hline L1CAM positive & & $3.78(1.69-7.61)$ & 0.002 & & $7.82(2.65-21.12)$ & 0.001 \\
\hline \multicolumn{7}{|l|}{ MMR-D } \\
\hline L1CAM negative (ref) & $33 / 127$ & - & & $16 / 126$ & - & \\
\hline \multicolumn{7}{|l|}{ B } \\
\hline \multirow[t]{2}{*}{ Age } & $112 / 452$ & & & $55 / 450$ & & \\
\hline & & 34.16 (10.16-117) & $<0.001$ & & $4.61(0.83-25.67)$ & 0.08 \\
\hline ProMisE & $112 / 452$ & & & $55 / 450$ & & \\
\hline p53 wt/NSMP (ref) & & - & & & - & \\
\hline POLE & & $0.84(0.28-1.95)$ & $<0.001$ & & $0.32(0.02-1.61)$ & 0.002 \\
\hline MMR-D & & $1.49(0.93-2.38)$ & & & $1.73(0.86-3.46)$ & \\
\hline p53 abn & & $3.18(1.71-5.97)$ & & & $3.97(1.74-9.31)$ & \\
\hline L1CAM status & $112 / 452$ & & & $55 / 450$ & & \\
\hline Negative (ref) & & - & & & - & \\
\hline
\end{tabular}

molecular subgroup tumours, which commonly overexpress L1CAM (Table 1). After hysterectomy, patients with p53 wt/NSMP, L1CAM-positive tumours were at similar risk for fatal outcome when compared to patients with p53 abn tumours (Table 3 and Fig. 2). These patients might benefit from the addition of adjuvant therapy, even if current ESMO criteria indicate a low-risk situation. Similarly, molecular testing is already well established to determine the need for adjuvant treatment in early-stage breast cancer patients. ${ }^{33,34}$

A similar negative prognostic effect of L1CAM expression in the other intermediate-risk group of MMR-D tumours was recently demonstrated; ${ }^{14,31}$ we observed a similar trend; however, statistical significance was not reached in our cohort (Table 2A); our study cohort is heterogeneous in stage, ESMO-risk group, 

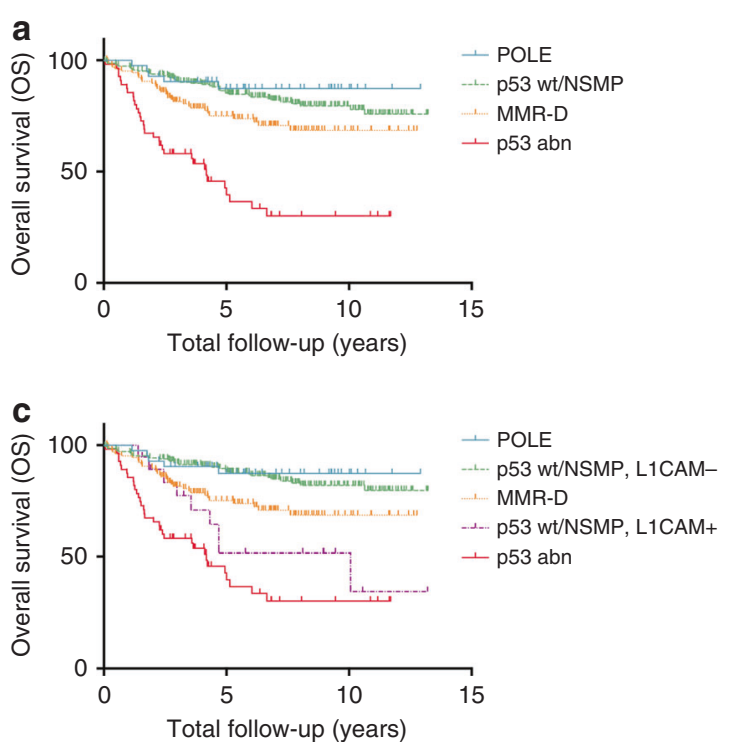
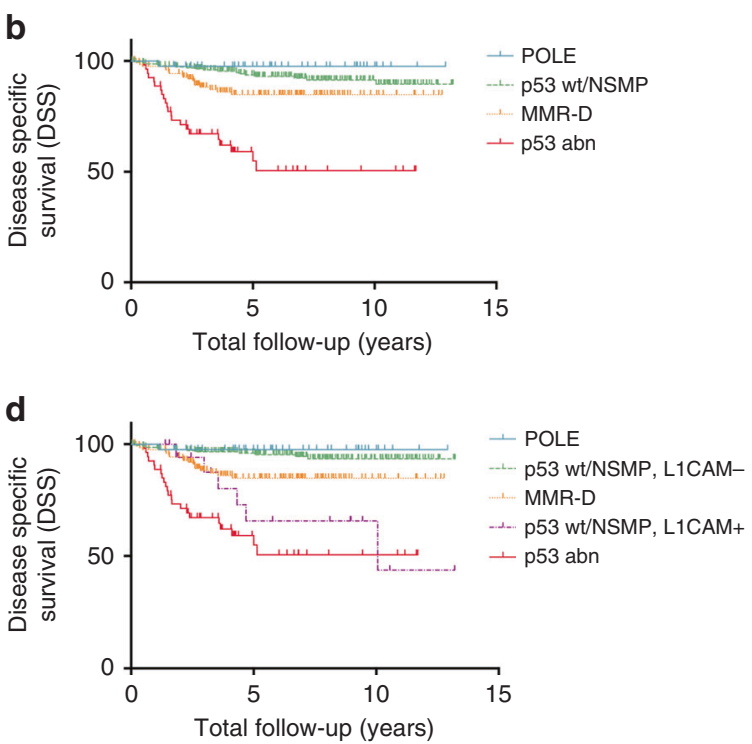

Fig. 2 Kaplan-Meier survival analysis considering ProMisE subgroups and L1CAM expression. OS and DSS of ProMisE subgroups before $(\mathbf{a}, \mathbf{b})$ and after (c and $\mathbf{d})$ stratifying for L1CAM status within the p53 wt/NSMP subgroup. a Five-year OS rates of $87.3 \%$ for POLE tumours, $85.2 \%$ for p53 wt/NSMP tumours, $75.0 \%$ for MMR-D tumours, and $39.6 \%$ for p53 abn tumours $(p<0.001)$. b Five-year DSS rates of $97.6 \%$ for POLE tumours, $93.6 \%$ for p53 wt/NSMP tumours, $84.7 \%$ for MMR-D tumours, and $54.8 \%$ for p53 abn tumours $(p<0.001)$ c Five-year OS rates of $87.3 \%$ for POLE tumours, $88.2 \%$ for p53 wt/NSMP, L1CAM negative tumours, 75.0\% for MMR-D tumours, 51.5\% for p53 wt/NSMP, L1CAM-positive tumours and $39.6 \%$ for $\mathrm{p} 53$ abn tumours $(p<0.001)$ d Five-year DSS rates of $97.6 \%$ for POLE tumours, $95.5 \%$ for p53 wt/NSMP, L1CAM negative tumours, $84.7 \%$ for MMR-D tumours, $65.5 \%$ for p53 wt/NSMP, L1CAM-positive tumours and 54.8\% for p53 abn tumours for disease-specific 5 -year survival $(p<0.001)$

Table 3. Univariate survival analysis (OS and DSS) considering ProMisE subgroups and L1CAM status within the p53 wt/NSMP subgroup

\begin{tabular}{|c|c|c|c|c|c|c|}
\hline ProMisE & $112 / 452$ & & & $55 / 450$ & & \\
\hline p53 wt/NSMP L1CAM- (ref) & & - & & & - & \\
\hline POLE & & $0.81(0.32-2.08)$ & $<0.001$ & & $0.46(0.06-3.58)$ & $<0.001$ \\
\hline MMR-D & & $2.07(1.27-3.37)$ & & & $2.99(1.40-6.40)$ & \\
\hline
\end{tabular}

treatment, and has relatively few L1CAM-positive tumours, which limited our power to study the prognostic significance of L1CAM in specific subgroups of tumours. If their finding is reproduced in other studies, it may further expand the potential of L1CAM IHC to refine the prognosis of intermediate-risk tumours.

While L1CAM-positive tumours clearly have worse outcomes, we support maintaining the original 4 TCGA/ProMisE tumour subgroups. We do not consider L1CAM-positive tumours as a distinct EC subtype, but instead as a prognostic subgroup within p53 wt/NSMP tumours and possibly MMR-D tumours. L1CAM expression seems to co-segregate with p53 abn status, and is therefore not a prognostic subgroup within this tumour subtype, and we are unaware of evidence to suggest L1CAM is prognostic within the POLE subtype. Restricting L1CAM IHC use to p53 wt/NSMP (and perhaps the MMR-D) subgroup tumours would provide economic advantage over performing L1CAM IHC on all cases.

L1CAM expression in experimental models has been shown to induce tumour cell migration, invasion, EMT, and chemo resistance. ${ }^{27,28}$ Several of these parameters can plausibly be linked to advanced stage and poor outcomes observed in L1CAMpositive tumours. In this context, a recent study has demonstrated that increased invasiveness of chemoresistant pancreatic cancer cells functionally depends on L1CAM. ${ }^{35}$ It is for these biological reasons that L1CAM is being explored as a therapeutic target in cancer. $^{17}$

The molecular basis for high L1CAM expression in some ECs remains unclear. The p53 wt subgroup comprises a group of EC which, at the time, had no prognostically significant mutation pattern. ${ }^{16}$ However, one mutation observed in up to $50 \%$ of this subgroup is activating mutations in CTNNB1 (encoding $\beta$-catenin). ${ }^{9}$ CTNNB1 mutations occur as part of a mutation module within these carcinomas together with mutations in KRAS and SOX17, all of which are known to activate Wnt-signalling in colon cancer. ${ }^{36-38}$ Activation of the "canonical" Wnt/ $\beta$-catenin pathway leads to stabilisation and accumulation of $\beta$-catenin within the nucleus, which is thought to induce cell proliferation and EMT. ${ }^{39-41}$ As described above, L1CAM is a target gene of $\beta$-catenin in colorectal cancer, and its transcription can be induced in EC cell lines by the EMT inducer Slug. ${ }^{41,42}$ We hypothesise that mutations of CTNNB1 in p53 wt/NSMP subgroup tumours could induce L1CAM expression and thereby promote more aggressive behaviour. CTNNB1 mutation is concentrated in p53 wt/NSMP tumours and predicts poor outcomes in EC, which raises the 


\begin{tabular}{|c|c|c|c|c|c|c|}
\hline & & OS & & & DSS & \\
\hline \multicolumn{7}{|l|}{ A } \\
\hline Age & $41 / 228$ & & & $16 / 227$ & & \\
\hline Negative (ref) & & - & & & - & \\
\hline Positive & & $2.43(0.99-5.41)$ & 0.052 & & $3.80(1.10-12.16)$ & 0.035 \\
\hline Tumour grade & $41 / 228$ & & & $16 / 227$ & & \\
\hline Grade 1 and 2 (ref) & & - & & & - & \\
\hline Grade 3 & & $2.70(1.00-6.40)$ & 0.049 & & $4.97(1.33-16.55)$ & 0.019 \\
\hline \multicolumn{7}{|l|}{ B } \\
\hline \multirow[t]{2}{*}{ Age } & $41 / 228$ & & & $16 / 227$ & & \\
\hline & & $1.05(1.02-1.08)$ & $<0.001$ & & $1.04(0.99-1.09)$ & 0.068 \\
\hline L1CAM & $41 / 228$ & & & $16 / 227$ & & \\
\hline Negative (ref) & & - & & & - & \\
\hline Positive & & $2.26(0.88-9.29)$ & 0.08 & & $4.03(1.11-13.74)$ & 0.035 \\
\hline Tumour grade & $41 / 228$ & & & $16 / 227$ & & \\
\hline Grade 1 and 2 (ref) & & - & & & - & \\
\hline Grade 3 & & $1.84(0.65-4.62)$ & 0.2 & & $2.73(0.75-8.85)$ & 0.1 \\
\hline Histology & $41 / 228$ & & & $16 / 227$ & & \\
\hline Endometrioid (ref) & & - & & & - & \\
\hline
\end{tabular}

possibility that CTNNB1 mutation analysis or $\beta$-catenin IHC could add additional prognostic information to the ProMisE molecular classifier by identifying tumours at higher risk for recurrence within the large p53 wt/NSMP subgroup. ${ }^{43,44}$ Further studies should investigate the correlation between CTNNB1 mutation and L1CAM expression in p53 wt/NSMP tumours to determine whether these variables are linked and, conclusively, whether to incorporate one or both markers into the ProMisE molecular classifier.

\section{CONCLUSION}

This study indicates that L1CAM expression status could add important prognostic information to the molecular classification of EC. L1CAM IHC was able to further stratify risk within the p53 wt/NSMP subgroup by identifying carcinomas at higher risk for fatal outcome. A strong correlation between mutation-type p53 immunostaining and L1CAM expression in EC was also confirmed. Taken together with previous studies, our results support adding L1CAM IHC as part of a simplified, clinically applicable molecular classifier for EC. Furthermore, we suggest investigating the role of CTNNB1 mutation status within the p53 wt/NSMP subgroup of EC and its correlation with aberrant L1CAM expression.

\section{ACKNOWLEDGEMENTS}

We are grateful for the work of Karen Greif, Friederike Grevenkamp and Anne-Kathrin Bunz. This research did not receive any specific grant from funding agencies in the public, commercial or not-for-profit sectors.

\section{AUTHORS CONTRIBUTIONS}

F.K.F.K.: conceptualisation, data analysis, writing original draft, visualisation; A.N.K.: editing of manuscript; F.K.: editing of manuscript; A.T.: data analysis; F.-A.T.: clinical data; A.S.: immunohistochemistry; C.B.G.: conceptualisation; D.G.H.: resources; B.K.: clinical data; S.Y.B.: clinical data, resources; J.N.M.: conceptualisation, resources; S.K.: Conceptualisation, supervision, project administration, editing of manuscript; All authors reviewed the manuscript.

\section{ADDITIONAL INFORMATION}

Supplementary information is available for this paper at https://doi.org/10.1038/ s41416-018-0187-6.

Competing interests: The authors declare no competing interests.

Ethical standards: Study approval was obtained from the Independent Ethics Committee of the University of Tübingen. All procedures were performed in accordance with the ethical standards of the institutional research committee and the Declaration of Helsinki. 
Availability of data and material: The datasets generated during and/or analysed during the current study are available from the corresponding author on reasonable request.

Note: This work is published under the standard license to publish agreement. After 12 months the work will become freely available and the license terms will switch to a Creative Commons Attribution 4.0 International (CC BY 4.0).

\section{REFERENCES}

1. Morice, P., Leary, A., Creutzberg, C., Abu-Rustum, N. \& Darai, E. Endometrial cancer. Lancet 387, 1094-1108 (2016).

2. Colombo, N. et al. ESMO-ESGO-ESTRO consensus conference on endometrial cancer: diagnosis, treatment and follow-up. Int. J. Gynecol. Cancer 26, 2-30 (2016).

3. Creutzberg, C. L. et al. Surgery and postoperative radiotherapy versus surgery alone for patients with stage- 1 endometrial carcinoma: multicentre randomised trial. Lancet 355, 1404-1411 (2000).

4. Fanning, J. Long-term survival of intermediate risk endometrial cancer (stage IG3, IC, II) treated with full lymphadenectomy and brachytherapy without teletherapy. Gynecol. Oncol. 82, 371-374 (2001).

5. Mariani, A. et al. Prospective assessment of lymphatic dissemination in endometrial cancer: a paradigm shift in surgical staging. Gynecol. Oncol. 109, 11-18 (2008).

6. Clarke, B. A. \& Gilks, C. B. Endometrial carcinoma: controversies in histopathological assessment of grade and tumour cell type. J. Clin. Pathol. 63, 410-415 (2010).

7. Grevenkamp, F. et al. Second opinion expert pathology in endometrial cancer: potential clinical implications. Int. J. Gynecol. Cancer 27, 289-296 (2017).

8. Chafe, S., Honore, L., Pearcey, R. \& Capstick, V. An analysis of the impact of pathology review in gynecologic cancer. Int. J. Radiat. Oncol. - Biol. - Phys. 48, 1433-1438 (2000).

9. Kandoth, C. et al. Integrated genomic characterization of endometrial carcinoma. Nature 497, 67-73 (2013).

10. Church, D. N. et al. DNA polymerase epsilon and delta exonuclease domain mutations in endometrial cancer. Hum. Mol. Genet. 22, 2820-2828 (2013).

11. Talhouk, A. et al. A clinically applicable molecular-based classification for endometrial cancers. Br. J. Cancer 113, 299-310 (2015).

12. Talhouk, A. et al. Confirmation of ProMisE: A simple, genomics-based clinical classifier for endometrial cancer. Cancer 123, 802-813 (2017).

13. Stelloo, E. et al. Refining prognosis and identifying targetable pathways for highrisk endometrial cancer; a TransPORTEC initiative. Mod. Pathol. 28, 836-844 (2015).

14. Stelloo, E. et al. Improved risk assessment by integrating molecular and clinicopathological factors in early-stage endometrial cancer-combined analysis of the PORTEC cohorts. Clin. Cancer Res. 22, 4215-4224 (2016).

15. Kommoss, S. et al. Final validation of the promise molecular classifier for endometrial carcinoma in a large population-based case series. Ann. Oncol. 29, 1180-1188 (2018).

16. McAlpine, J., Leon-Castillo, A. \& Bosse, T. The rise of a novel classification system for endometrial carcinoma; integration of molecular subclasses. J. Pathol. 244, 538-549 (2018).

17. Altevogt, P., Doberstein, K., Fogel, M. L1CAM in human cancer. Int. J. Cancer 138, 1565-1576 (2015).

18. Fogel, M. et al. L1 expression as a predictor of progression and survival in patients with uterine and ovarian carcinomas. Lancet 362, 869-875 (2003).

19. Zeimet, A. G. et al. L1CAM in early-stage type I endometrial cancer: results of a large multicenter evaluation. J. Natl Cancer Inst. 105, 1142-1150 (2013).

20. Bosse, T. et al. L1 cell adhesion molecule is a strong predictor for distant recurrence and overall survival in early stage endometrial cancer: pooled PORTEC trial results. Eur. J. Cancer 50, 2602-2610 (2014).
21. Dellinger, T. H. et al. L1CAM is an independent predictor of poor survival in endometrial cancer-an analysis of The Cancer Genome Atlas (TCGA). Gynecol. Oncol. 141, 336-340 (2016).

22. Van der Putten, L. J. et al. L1CAM expression in endometrial carcinomas: an ENITEC collaboration study. Br. J. Cancer 115, 716-724 (2016).

23. Kommoss, F. et al. L1CAM: amending the "low-risk" category in endometrial carcinoma. J. Cancer Res. Clin. Oncol. 143, 255-262 (2016).

24. Kiefel, H., Pfeifer, M., Bondong, S., Hazin, J. \& Altevogt, P. Linking L1CAM-mediated signaling to NF-kappaB activation. Trends Mol. Med. 17, 178-187 (2011).

25. Shtutman, M., Levina, E., Ohouo, P., Baig, M. \& Roninson, I. B. Cell adhesion molecule L1 disrupts E-cadherin-containing adherens junctions and increases scattering and motility of MCF7 breast carcinoma cells. Cancer Res. 66, 11370-11380 (2006)

26. Tischler, V. et al. L1CAM protein expression is associated with poor prognosis in non-small cell lung cancer. Mol. Cancer 10, 127 (2011).

27. Kiefel, H. et al. L1CAM: a major driver for tumor cell invasion and motility. Cell Adh. Migr. 6, 374-384 (2012).

28. Chen, J., Gao, F. \& Liu, N. L1CAM promotes epithelial to mesenchymal transition and formation of cancer initiating cells in human endometrial cancer. Exp. Ther. Med. 15, 2792-2797 (2018).

29. Huszar, M. et al. Up-regulation of L1CAM is linked to loss of hormone receptors and E-cadherin in aggressive subtypes of endometrial carcinomas. J. Pathol. 220, 551-561 (2010).

30. Colas, E. et al. The EMT signaling pathways in endometrial carcinoma. Clin. Transl. Oncol. 14, 715-720 (2012).

31. Karnezis, A. N. et al. Evaluation of endometrial carcinoma prognostic immunohistochemistry markers in the context of molecular classification. J. Pathol. Clin. Res. 3, 279-293 (2017).

32. Van Gool, I. C. et al. Prognostic significance of L1CAM expression and its association with mutant p53 expression in high-risk endometrial cancer. Mod. Pathol. 29, 174-181 (2016).

33. van 't Veer, L. J. et al. Gene expression profiling predicts clinical outcome of breast cancer. Nature 415, 530 (2002).

34. Paik, S. et al. Gene expression and benefit of chemotherapy in women with nodenegative, estrogen receptor-positive breast cancer. J. Clin. Oncol. 24, 3726-3734 (2006).

35. Lund, K. et al. Slug-dependent upregulation of L1CAM is responsible for the increased invasion potential of pancreatic cancer cells following long-term 5-FU treatment. PLOS ONE 10, e0123684 (2015).

36. Sinner, D. et al. Sox 17 and Sox 4 differentially regulate beta-catenin/T-cell factor activity and proliferation of colon carcinoma cells. Mol. Cell. Biol. 27, 7802-7815 (2007).

37. Zorn, A. M. et al. Regulation of Wnt signaling by sox proteins: XSox $17 \mathrm{a} / \mathrm{b}$ and XSox3 physically interact with b-catenin. Mol. Cell 4, 487-498 (1999).

38. Li, J., Mizukami, Y., Zhang, X., Jo, W.-S. \& Chung, D. C. Oncogenic K-ras stimulates Wnt signaling in colon cancer through inhibition of GSK-3 $\beta$. Gastroenterology 128, 1907-1918 (2005).

39. Kalluri, R. \& Weinberg, R. A. The basics of epithelial-mesenchymal transition. J. Clin. Invest. 119, 1420-1428 (2009).

40. Raveh, S., Gavert, N. \& Ben-Ze'ev, A. L1 cell adhesion molecule (L1CAM) in invasive tumors. Cancer Lett. 282, 137-145 (2009).

41. Gavert, N. et al. L1, a novel target of beta-catenin signaling, transforms cells and is expressed at the invasive front of colon cancers. J. Cell Biol. 168, 633-642 (2005).

42. Pfeifer, M. et al. L1CAM expression in endometrial carcinomas is regulated by usage of two different promoter regions. BMC Mol. Biol. 11, 64 (2010).

43. Kurnit, K. et al. CTNNB1 (beta-catenin) mutation identifies low grade, early stage endometrial cancer patients at increased risk of recurrence. Mod. Pathol. 30, 1032-1041 (2017)

44. Liu Y., et al. Clinical significance of CTNNB1 mutation and Wnt pathway activation in endometrioid endometrial carcinoma. J. Natl. Cancer. Inst. 106, dju245 (2014) 